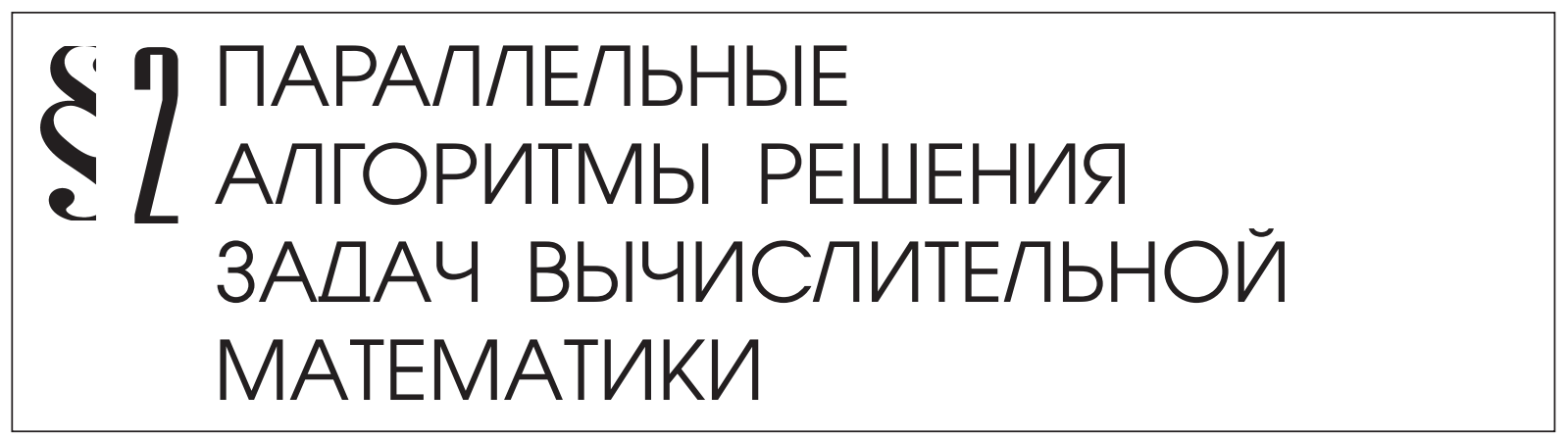

Клименко А. Б., Троценко Р. В.

\title{
РЕШЕНИЕ ЗАДАЧИ ОПТИМИЗАЦИИ РЕСУРСОВ И ПЛАНИРОВАНИЯ ВЫЧИСЛЕНИЙ С ИСПОЛЬЗОВАНИЕМ ПАРАЛЛЕЛЬНОЙ ИМИТАЦИИ ОТЖИГА
}

\begin{abstract}
Аннотация: Для решения задачи оптимизачии используемых ресурсов и планирования вычислений в настоящее время успешно используются различные метаэвристики, в частности, метод имитации отжига. Имитация отжига - метод последовательный, что затрудняет его распараллеливание, однако, в последнее время были разработаны различные способы распараллеливания с цельюулучшения качества получаемых решений и времени работы алгоритма.Предметом исследования являются методы распараллеливания имитации отжига, в частности, метод независимыхзапусков с синхронизацией и асинхронный. Вкачестве примера реализации имитации отжигавыбран метод стемпературной схемой "тушения", как ниболее быстрый. Проведен аналитический обзор методов распараллеливания имитации отжига с выделением наиболее перспективных, для которых проведена серия вычислительных экспериментов с использованием. Научная новизна заключается в обнаружении новыхзависимостей и тенденций, ранее не описанных в подобных исследованиях: для параллельной имитации отжига с синхронизацией поднят вопрос существования зависимости качества получаемого решения не только от количества вычисляющих устройств, но и от частоты обмена решениями. Для параллельнойй асинхронной имитации отжига прослеживается тенденция улучшения решений сувеличением количества вычисляющих устройств, тогда как для синхронной имитации отжига нельзя вести речь об однозначном улучшении и зависимости только от числа вычисляющих устройств. Ключевые слова: имитация отжига, параллельный алгоритм, оптимизация, вычислительная система, оптимизация ресурсов, параллельные вычисления, распределение ресурсов, метаэвристика, методы распараллеливания, планирование вычислений
\end{abstract}

В настоящее время одним из многообещающих направлений развития вычислительной техники является построение многопроцессорных комплексов и систем, а также 
grid-систем и систем распределенных вычислений, в том числе, узкоспециализированных. В процессе планирования таких систем одним из актуальных вопросов является удешевление суммарной стоимости системы, но при условии, чтобы поставленные перед ней задачи успешно решались в отведенные сроки. Задача оптимизации используемых ресурсов и планирования вычислений не нова, и первые работы, освящающие вопрос, появились в 60-х годах 20 столетия как расширение возможностей методов критического пути и PERT [1-3]. В дальнейшем, на территории России тема в аспекте формирования ресурсов многопроцессорных вычислительных систем была продолжена А.Б. Барским[4].

В общем виде задача формулируется следующим образом[4]: для заданного комплекса информационно и по управлению взаимосвязанных задач, заданной архитектуры

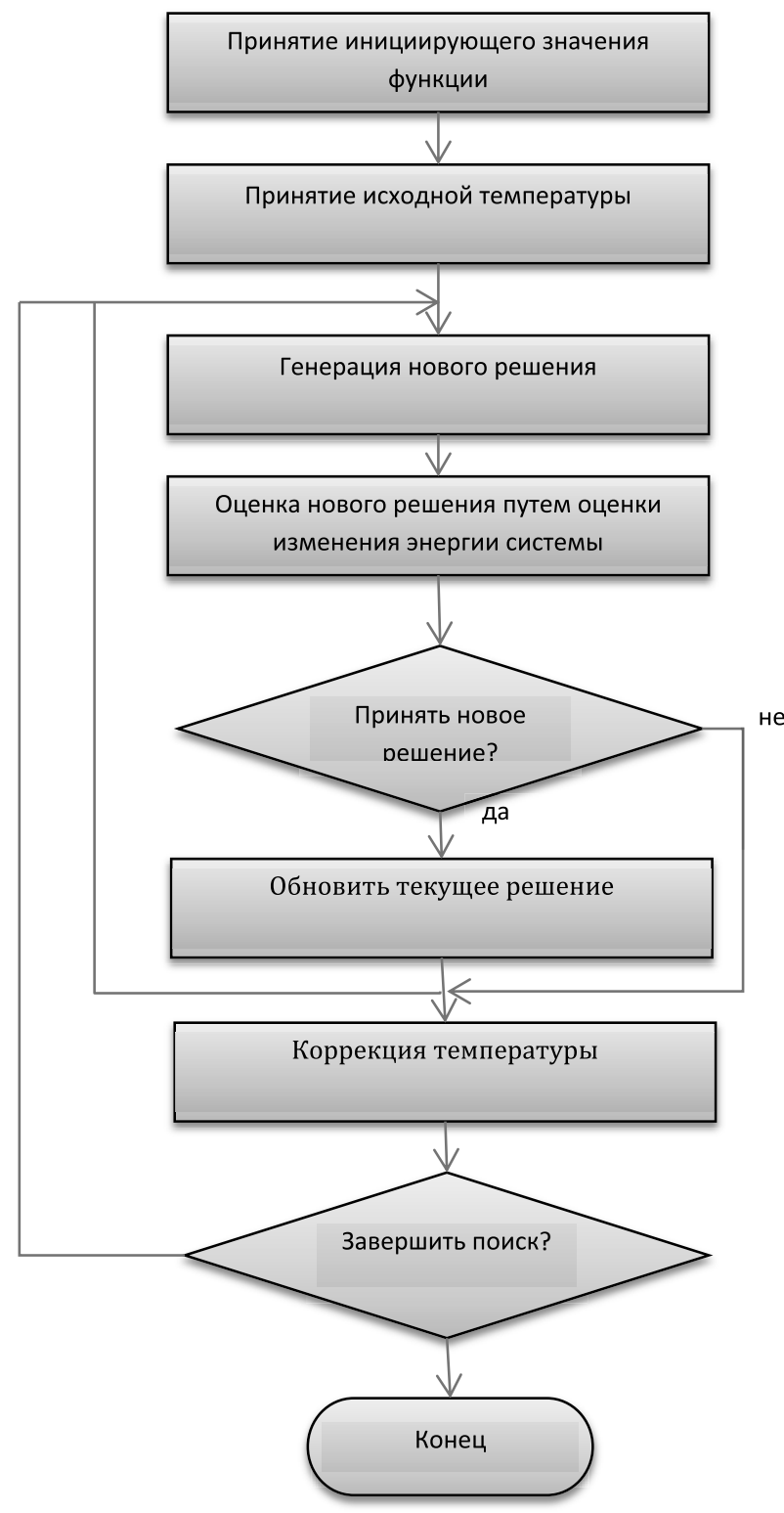

Рисунок 1. - Общая схема метода имитации отжига. вычислительной системы, а также заданного ограничения на допустимое время вычислительного процесса выбрать комплектацию ВС минимальной стоимости. Под стоимостью понимают вес каждого типа процессоров, который необходимо учитывать при выборе оптимальной комплектации.

В работе [4] был предложен точный метод решения задачи комплектации вычислительной системы минимальной стоимости, который, однако, может занять длительное время, учитывая принадлежность задачи к классу NP-сложных.

Необходимость решения подобных задач за приемлемое время стала обоснованием использования сперва эвристических методов, затем и метаэвристических. В свою очередь, использование многопроцессорных вычислительных систем и комплексов позволяет произвести улучшение результатов работы метаэвристик в аспектах затрачиваемого времени и качества получаемых решений.

Семейство алгоритмов имитации отжига (ИО) являются средством, позволяющим получать хорошие результаты для NP-сложных задач большой размерности благодаря возможности выхода из локальных оптимумов [5,6]. В общем виде алгоритм ИО может быть представлен, как показано на рис. 1. 
Алгоритмы ИО являются итерационными и последовательными, что затрудняет их распараллеливание. Тем не менее, были разработаны методы распараллеливания имитации отжига, позволившие значительно улучшить скорость и точность метода[7].

В настоящее время известны и используются на практике следующие методы распараллеливания ИО:

- независимые запуски (Multiple Independent Runs), с синхронизацией или асинхронные;

- с разбиением пространства решений на области;

- на основе декомпозиции целевой функции;

- распараллеливание на уровне цепей Маркова внутри температурной итерации.

Метод независимых параллельных запусков заключается в следующем: на множестве процессорных устройств (ПУ) производится запуск алгоритмов ИО, возможно, с различными точками инициализации вычислений, с выбором наилучшего решения по завершении. Независимые параллельные запуски с синхронизацией предполагают периодический обмен текущими решениями между ПУ с выбором наилучшей точки для продолжения вычислений. На рис. 2. и рис.3 представлены асинхронный MIR и MIR с синхронизацией.

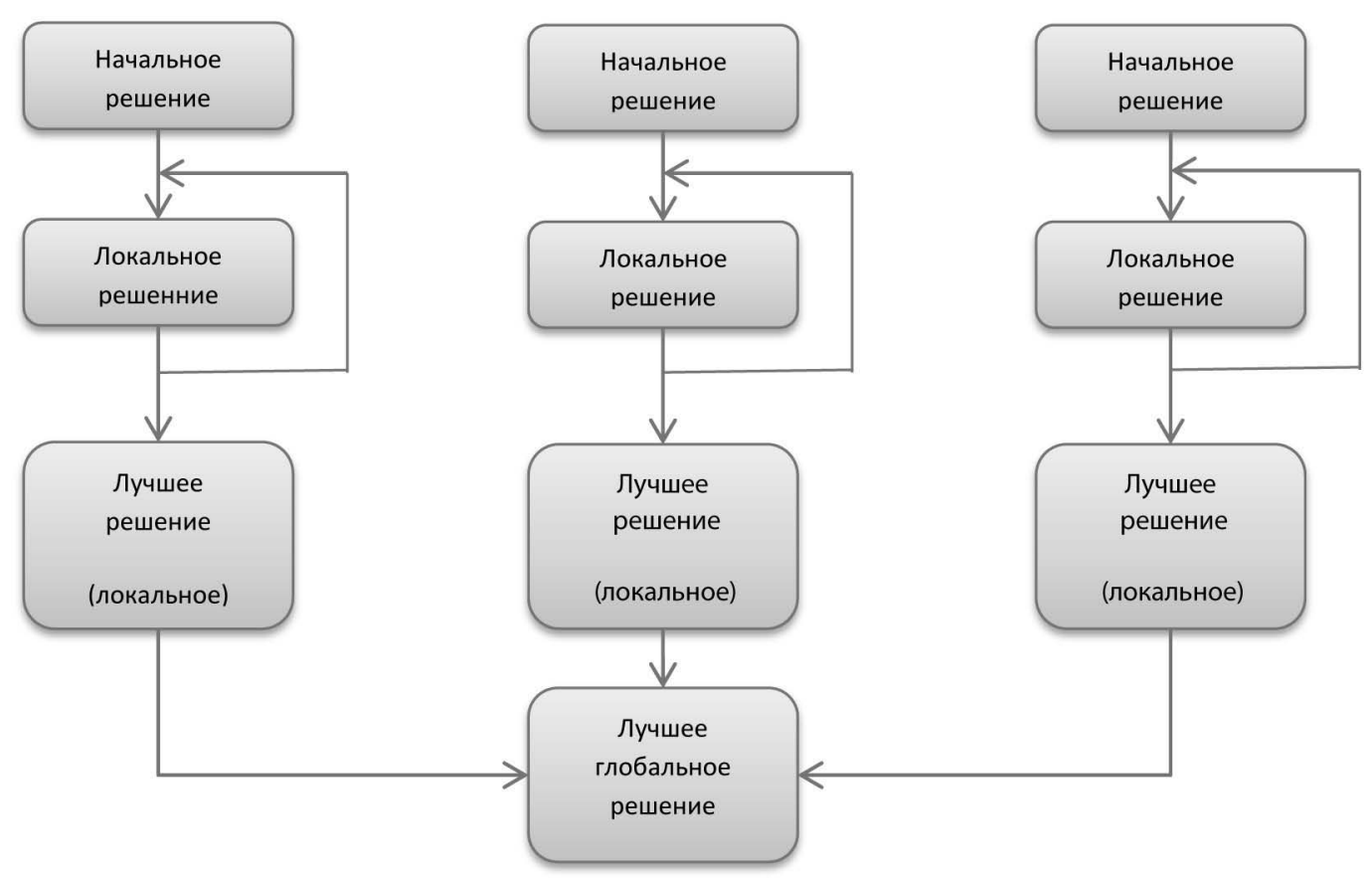

Рисунок 2 - Асинхронный MIR. 


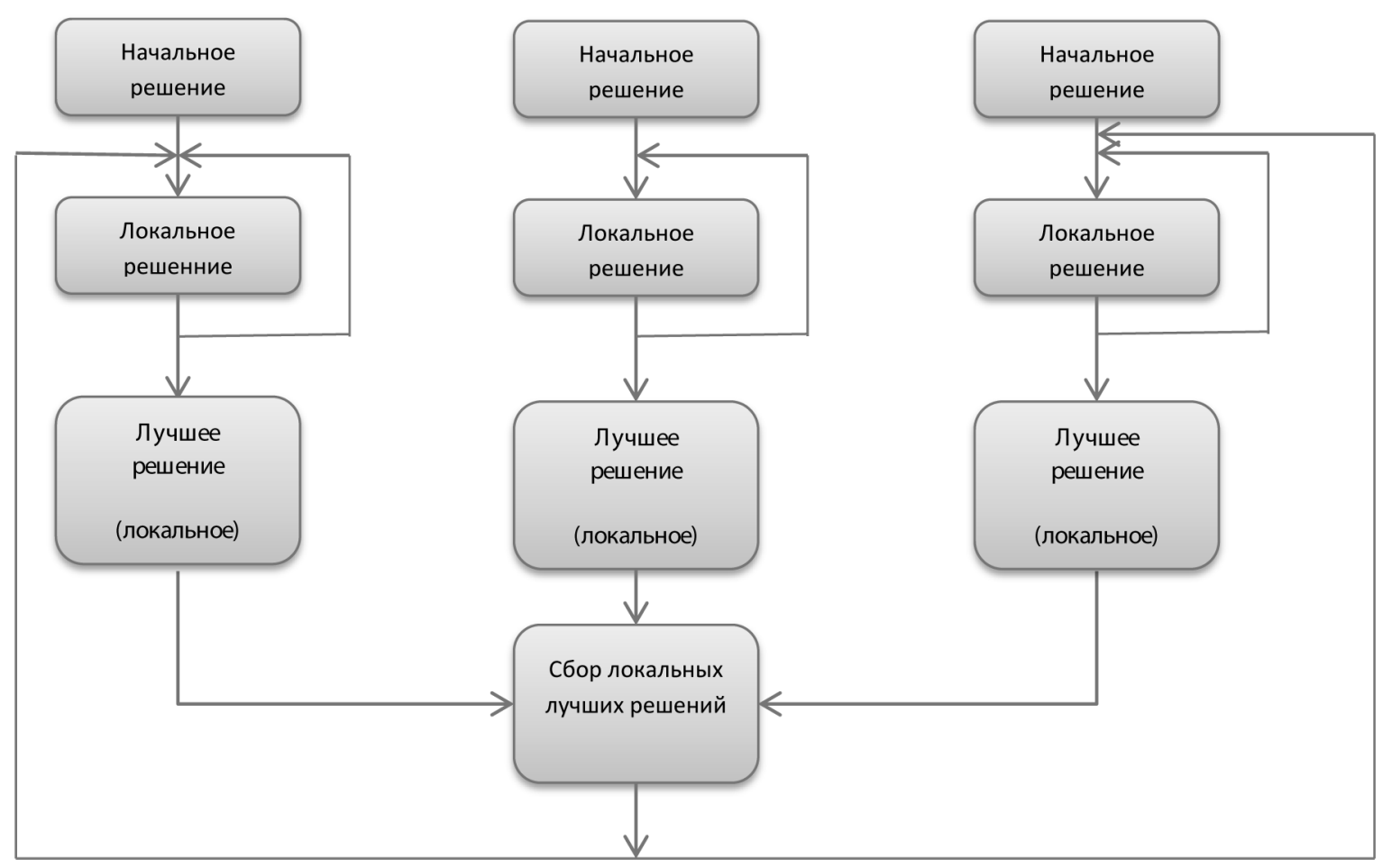

Трансляция лучшего решение в качестве нового локального

Рисунок 3 - MIR с синхронизацией

Метод, в основу которого положена декомпозиция целевой функции, является проблемно-ориентированным и подходит для целевых функций вида:

$$
F\left(x_{1}, x_{2}, \ldots x_{\mathrm{n}}\right)=F\left(x_{1}\right)+F\left(x_{2}\right)+\ldots+F\left(x_{\mathrm{n}}\right) \text {. }
$$

Примером задачи, для которой применялся такой метод распараллеливания, является задача размещения транзисторов на интегральной схеме, поскольку если в качестве целевой рассматривать количество перекрывающихся элементов, то оно может быть посчитано как сумма перекрывающихся элементов отдельных участков интегральной схемы. Однако, если в качестве ЦФ выбираются функции, которые затруднительно представить в виде (1), то применение данного метода распараллеливания становится невозможным.

Метод, использующий разбиение пространства решений на области, является универсальным, но при его использовании необходимо отслеживать попадание генерируемых решений в отведенную им область.

Последний метод с распараллеливанием марковских цепей интересен тем, что распараллеливание предполагается внутри одного температурного уровня. Пример распараллеливания приведен на рис. 4. 


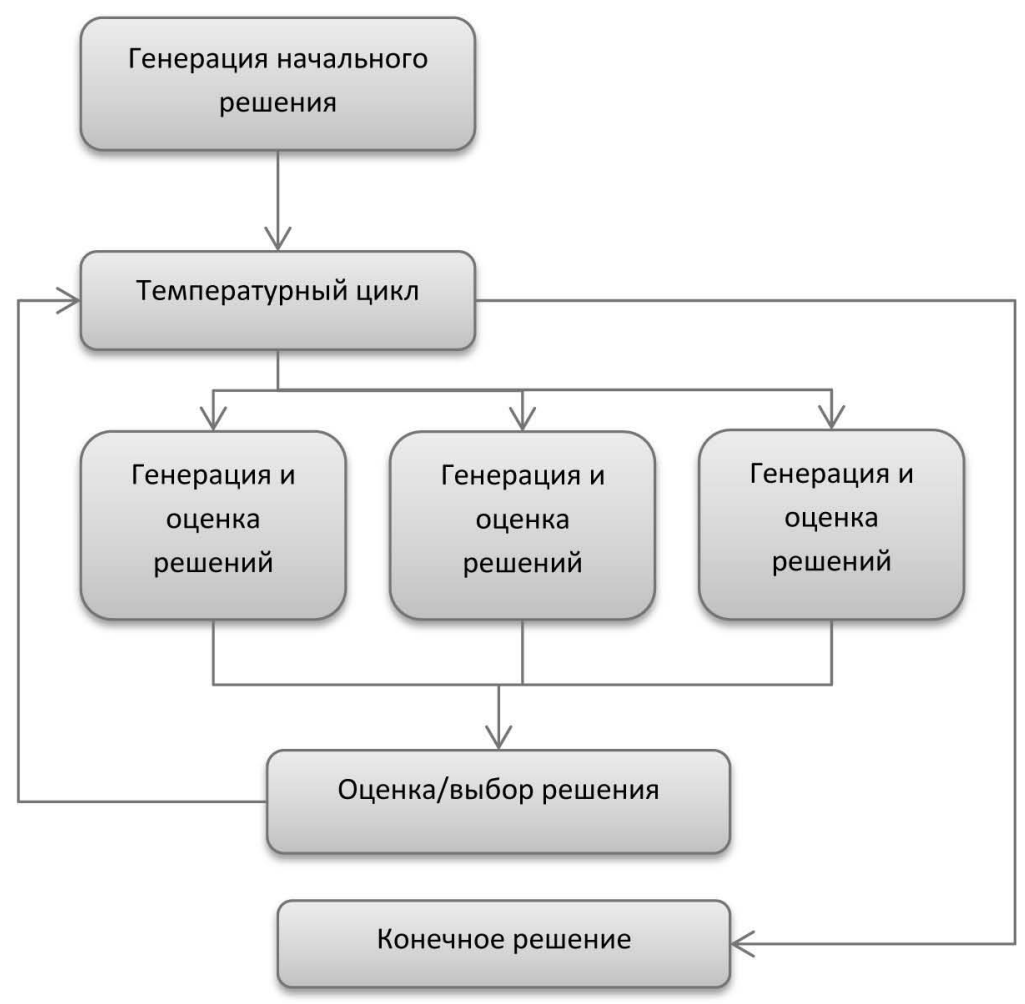

Рисунок 4 - Распараллеливание на уровне марковских цепей.

Для дальнейшего синтеза алгоритма решения задачи оптимизации используемых ресурсов и планирования вычислений с использованием параллельной имитации отжига будем опираться на допущение о том, что целесообразно придерживаться такой стратегии закрепления ресурсов за работами, чтобы минимизировать простои оборудования. Иными словами, мы допускаем использование эвристик, позволяющих строить расписания с минимизацией простоев/времени выполнения набора задач. Вэтом случае поиск при помощи ИО производится в пространстве решений, представляющих собой разнообразные комбинации ПУ, каждый из которых может обладать собственной производительностью. Схематично алгоритм ИО для решения задачи оптимизации используемых ресурсов и планирования вычислений может быть представлен следующим образом.

1. Задать исходные: температуру, комбинацию ВУ.

2. Составить расписание для выбранной комбинации.

3. Если время выполнения всех работ превышает заданное, то сформировать новое решение и перейти к п.2.

4. Генерация нового решения: выбираем случайным образом новую комбинацию.

5. Строим расписание для нового решения.

6. Если время выполнения всех работ превышает заданное, то перейти к п.4, в противном случае: сравниваем полученное новое решение с предыдущим. Если новое решение лучше, то в качестве текущего решения принимаем его. Если хуже, то принимаем его в 
качестве текущего с вероятностью $e^{-\Delta E / t}$, где $\Delta E$ - изменение энергии.

7. Пока не произведено заданное количество итераций, перейти к п.4.

8. Пока температура >0, понизить температуру и перейти к п.4.

Для проведения эксперимента были сформированы следующие входные данные: граф задач (20 вершин с трудоемкостями, заданными случайным образом), множество доступных ПУ с производительностями, заданными случайным образом мощностью 15. В качестве закона понижения температуры была выбрана схема «тушения», описываемая выражением $T_{k}=c T_{k-1}$ где коэффициент $c=0.8$. Была проведена серия тестов для параллельной ИО с синхронизацией для различных интервалов обменов между ПУ. Результаты трассировки получаемых решений (стоимостей системы) приведены ниже (рис. 5-8).

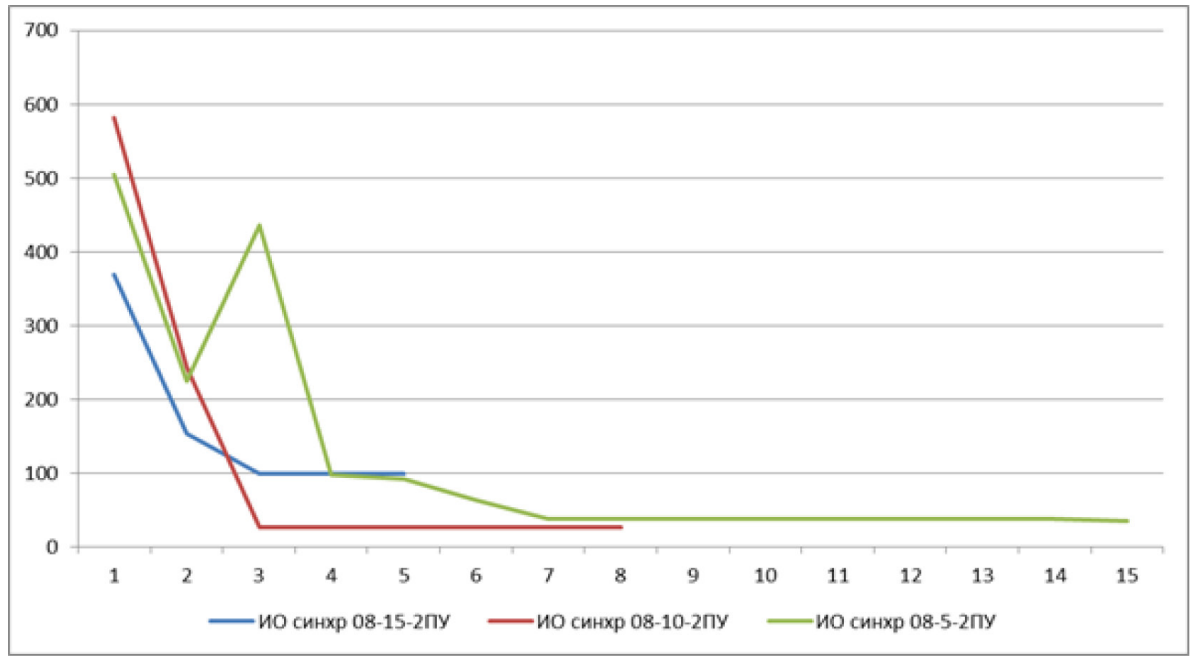

Рисунок 5 - Результаты трассировки решений для 2 ПУ.

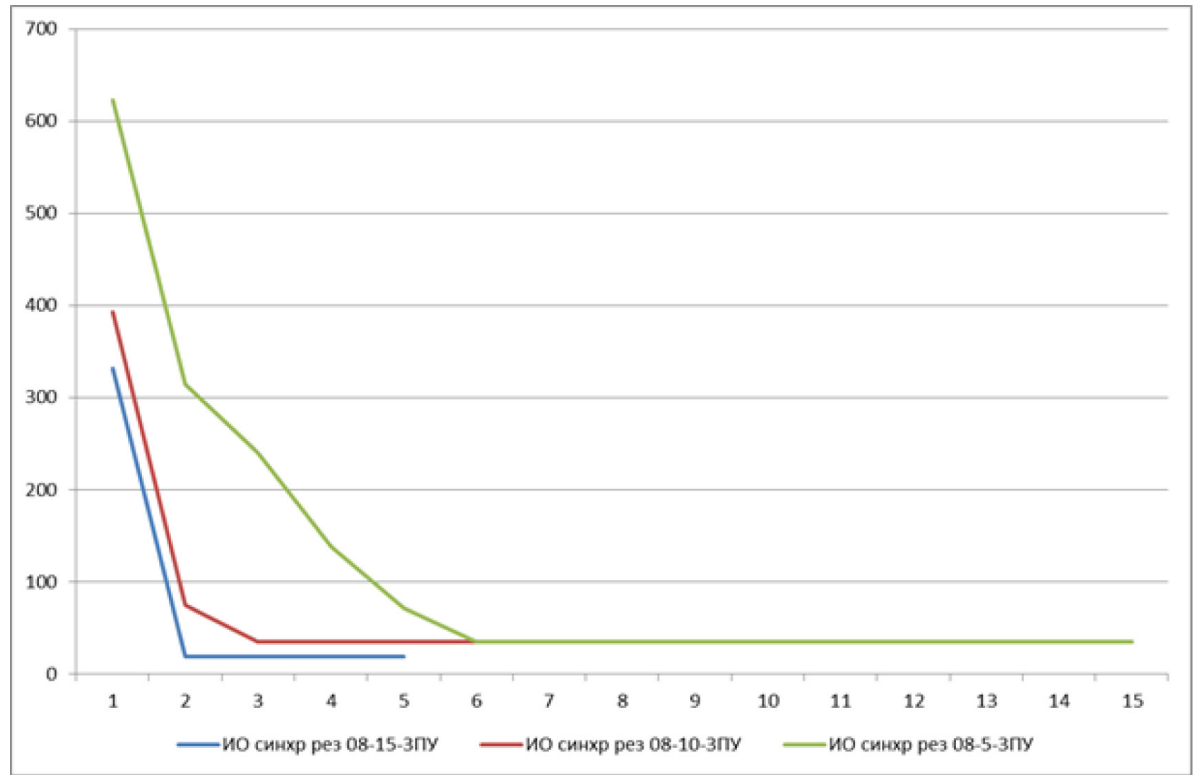

Рисунок 6 - Результаты трассировки решений для 3 Пу 


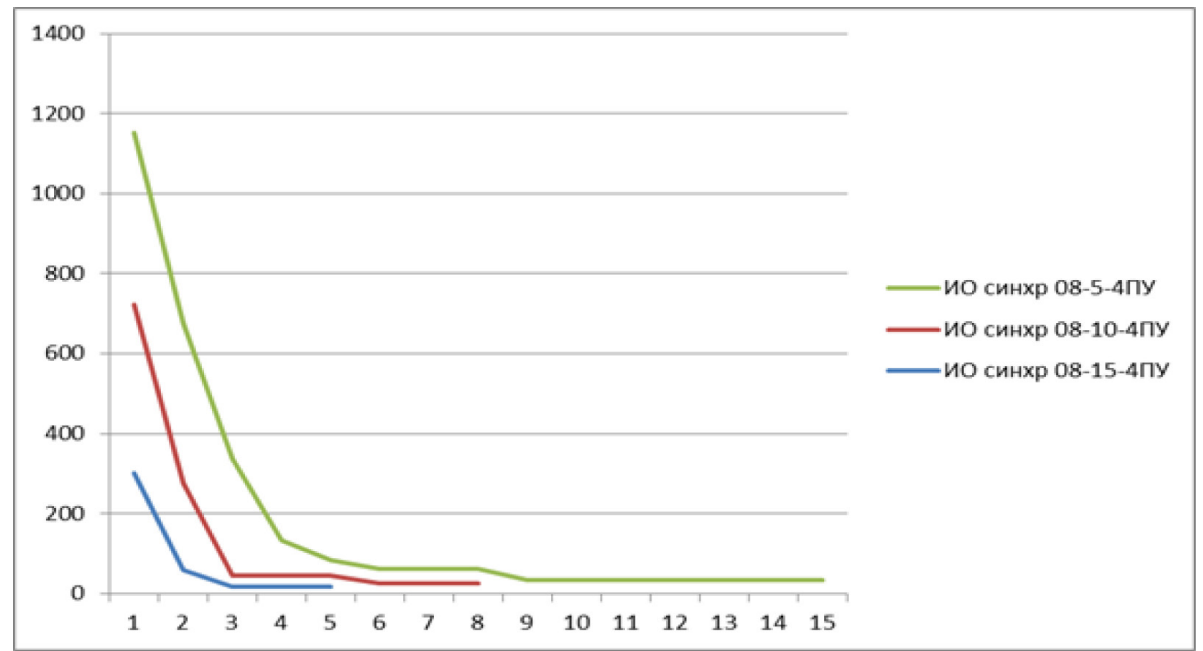

Рисунок 7 - Результаты трассировки решений для 4 ПУ.

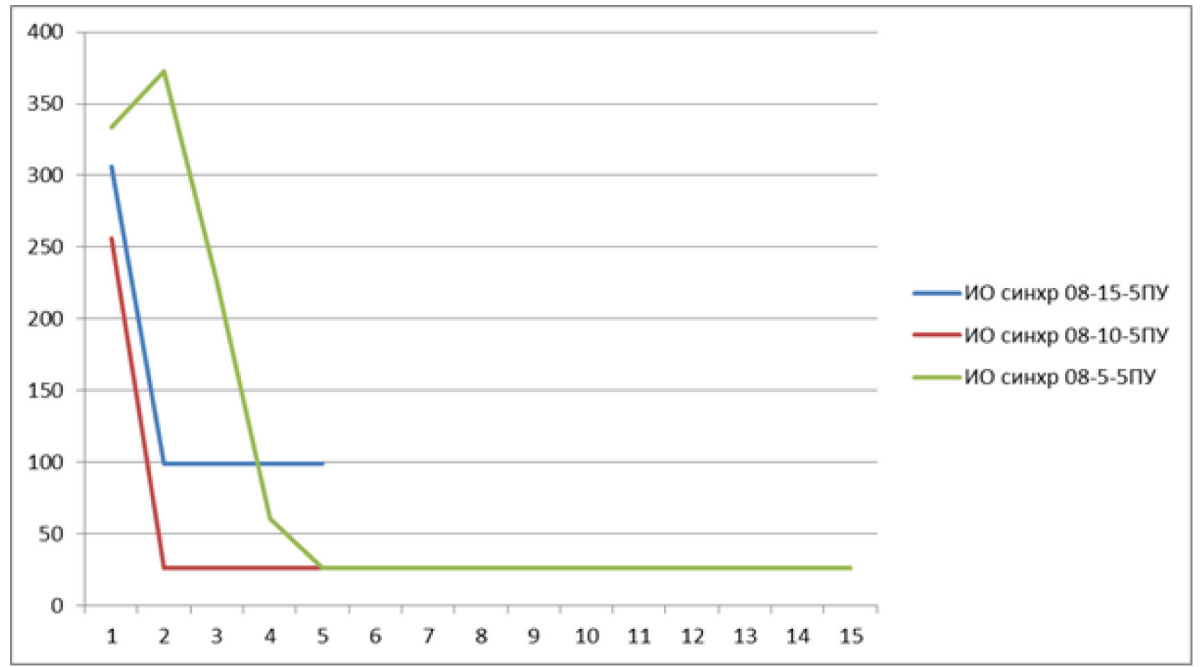

Рисунок 8 - Результаты трассировки решений для 5 Пу.

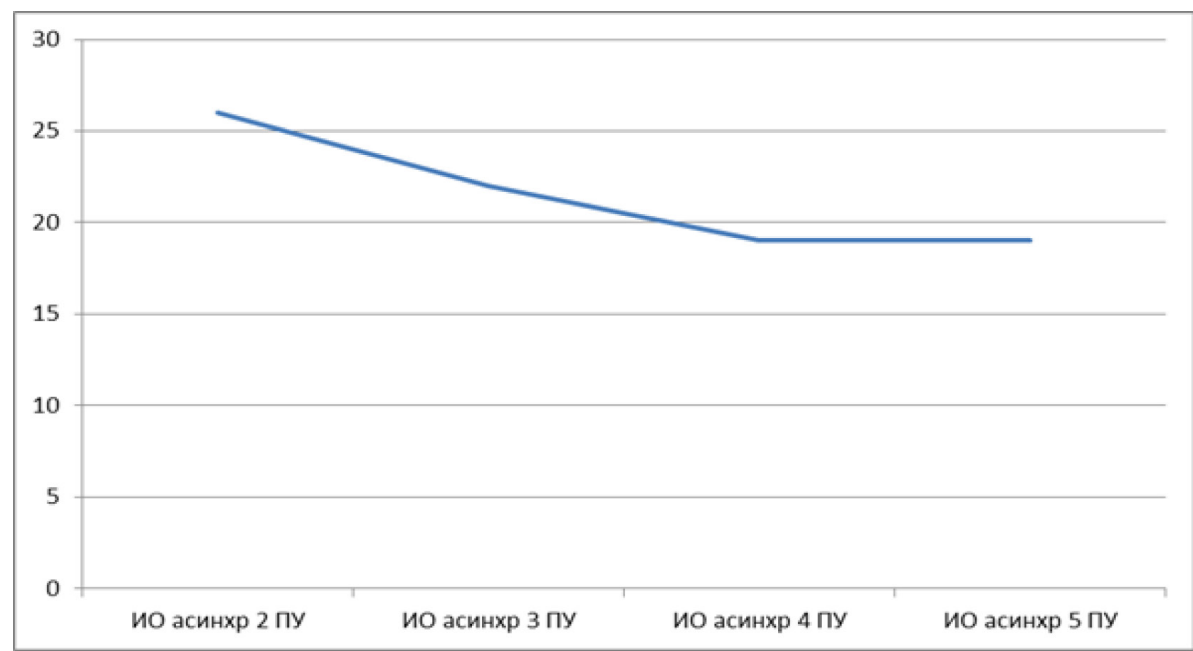

Рисунок 9 - Результат вычислений при асинхронном распараллеливании с выбором лучшего результата. 
Полученные в ходе эксперимента результаты позволяют сделать следующие выводы:

- Результат, получаемый в результате работы параллельного алгоритма ИО, также как и результат работы последовательной ИО, зависит от точки инициализации вычислений и не застрахован от попадания в локальные минимумы (это особенно актуально для температурных схем «тушения») независимо от количества параллельно работающих ПУ.

- Для распараллеливания ИО с синхронизацией наблюдается зависимость разброса получаемых решений от частоты обмена результатами между ПУ, при этом увеличение количества ПУ не всегда приводит к улучшению качества результата. Вопрос оптимизации интервалов обмена подлежит дальнейшей проработке.

- Для параллельной асинхронной ИО с выбором лучшего решения по окончании вычислений прослеживается наглядная тенденция улучшения качества результата с увеличением количества ПУ.

В заключение следует отметить, что результаты проведенного эксперимента, несомненно, имеют предварительный характер, и что для получения окончательного заключения требуется проведение более глубоких исследований параллельной ИО в свете решения задачи формирования ресурсов.

\section{Библиография :}

1. McGee A. A. and Markarian M. D. Optimum Allocation of Research (Engineering Manpower within a Multi-Project Organizational Structures). IRE Trans. Engng. Manag., v. 9, №. 3, 1962.

2. Levy F. E., Thompson G., Wiest J. Multiship, Multishop, Workloadsmoothing Program, Naval Research, Logistics Quarterly, v. 9, No. 1, 1962.

3. Fey C.F. Least Cost Estimating and Scheduling with Limited Resources. Abstract. Recent Advances Math. Programm, 1963.

4. Барский А.Б. Параллельные процессы в вычислительных системах: планирование и организация. - М.: Радио и связь, 1990.

5. F. Busetti, Simulated annealing overview. 2003. (http://citeseer.uark.edu:8080/citeseerx/viewdoc/summary;jsessi onid=9F958F3AD8ACB341A84315A737883592?doi=10.1.1.66.5018)

6. L.Ingber, Simulated annealing: practice versus theory. 1993. (http://citeseer.uark.edu:8080/citeseerx/viewdoc/summary?doi=10.1.1.15.1046)

7. Crainic T.G., Toulouse M. Parallel Metaheuristics. In T. G. Crainic and G. Laporte, editors, Fleet Management and Logistics, pages 205-25 1, 1998. Kluwer Academic Publishers.

\section{References:}

1. MsGee A. A. and Markarian M. D. Optimum Allocation of Research (Engineering Manpower within a Multi-Project Organizational Structures). IRE Trans. Engng. Manag., v. 9, №. 3, 1962. 
2. Levy F. E., Thompson G., Wiest J. Multiship, Multishop, Workloadsmoothing Program, Naval Research, Logistics Quarterly, v. 9, №. 1, 1962.

3. Fey S.F. Least Cost Estimating and Scheduling with Limited Resources. Abstract. Recent Advances Math. Programm, 1963.

4. Barskii A.B. Parallel'nye protsessy v vychislitel'nykh sistemakh: planirovanie i organizatsiya.

- M.: Radio i svyaz', 1990.

5. F. Busetti, Simulated annealing overview. 2003. (http://citeseer.uark.edu:8080/citeseerx/viewdoc/summary;jsessi onid=9F958F3AD8ACB341A84315A737883592? $\mathrm{doi}=10.1 .1 .66 .5018$ )

6. L.Ingber, Simulated annealing: practice versus theory.1993.

(http://citeseer.uark.edu:8080/citeseerx/viewdoc/summary?doi=10.1.1.15.1046)

7. Crainic T.G., Toulouse M. Parallel Metaheuristics. In T. G. Crainic and G. Laporte, editors, Fleet Management and Logistics, pages 205-25 1, 1998. Kluwer Academic Publishers. 